\title{
Magnetospheric Convection: From Mesoscale Flows To Microscale Instabilities
}

\author{
Aleksandr Y. Ukhorskiy ( $\nabla$ ukhorskiy@jhuapl.edu ) \\ Johns Hopkins University Applied Physics Laboratory \\ Kareem A. Sorathia \\ Johns Hopkins University Applied Physics Laboratory \\ Viacheslav G. Merkin \\ Johns Hopkins University Applied Physics Laboratory \\ Chris Crabtree \\ Naval Research Laboratory \\ Alex C. Fletcher \\ Naval Research Laboratory \\ David M. Malaspina \\ University of Colorado \\ Steven J. Schwartz \\ University of Colorado
}

\section{Research Article}

Keywords: magnetotail, plasma, kinetic, dipolarization

Posted Date: December 2nd, 2021

DOI: https://doi.org/10.21203/rs.3.rs-1112322/v1

License: (c) (i) This work is licensed under a Creative Commons Attribution 4.0 International License.

Read Full License 


\title{
Magnetospheric Convection: From Mesoscale Flows to Microscale Instabilities
}

\author{
Aleksandr Y. Ukhorskiy ${ }^{1,2, *}$, Kareem A. Sorathia ${ }^{1}$, Viacheslav G. Merkin ${ }^{1}$, Chris Crabtree $^{3}$, \\ Alex C. Fletcher ${ }^{3}$, David M. Malaspina ${ }^{4,5}$, and Steven J. Schwartz ${ }^{5,6}$
}

${ }^{1}$ SES, Johns Hopkins University Applied Physics Laboratory, Laurel, 20723, USA

${ }^{2}$ Physics Department, Dartmouth College, Hanover, 03755, USA

${ }^{3}$ Plasma Physics Division, Naval Research Laboratory, Washington, DC, 20375, USA

${ }^{4}$ Astrophysical and Planetary Sciences Department, University of Colorado, Boulder, CO, 80303, USA

${ }^{5}$ Laboratory for Atmospheric and Space Physics, University of Colorado, Boulder, CO, 80303, USA

${ }^{6}$ Emeritus, Blackett Laboratory, Imperial College London, London SW7 2AZ, UK

*ukhorskiy@jhuapl.edu

\begin{abstract}
Plasma convection in the Earth's magnetosphere from the distant magnetotail to the inner magnetosphere occurs largely in the form of mesoscale flows, i.e., discrete enhancements in the plasma flow with sharp dipolarizations of magnetic field. Recent spacecraft observations suggest that the dipolarization flows are associated with a wide range of kinetic processes such as kinetic Alfvén waves, whistler chorus waves, and nonlinear time-domain structures. In this paper we explore how mesoscale dipolarization flows produce suprathermal electron instabilities, thus providing free energy for the generation of the observed kinetic waves and structures. We employ three-dimensional test-particle simulations of electron dynamics one-way-coupled to a global magnetospheric model. The simulations show a rapid growth of interchanging regions of parallel and perpendicular electron temperature anisotropies distributed along the magnetic terrain formed around the dipolarization flows. Unencumbered in test-particle simulations, a rapid growth of velocity-space anisotropies in the collisionless magnetotail plasma is expected to be curbed by the generation of plasma waves. The results are compared with in situ observations of an isolated dipolarization flow at one of the spacecraft of the Magnetospheric Multiscale Mission, that show strong VLF wave activity alternating between broad-band wave activity and whistler waves. With estimated spatial extent being similar to the characteristic size of temperature anisotropy patches in our test-particle simulations, the observed bursts of VLF wave activity are likely to be produced by the parallel and perpendicular electron energy anisotropies driven by the dipolarization flow, as suggested by our results.
\end{abstract}

\section{Introduction}

Much of dynamics across Earth's magnetosphere-ionosphere system is powered by a highly dynamic flow of the solar wind permeated by the interplanetary magnetic field (IMF). The solar wind-magnetosphere coupling is the strongest during intervals of the southward IMF, when magnetic reconnection on the subsolar magnetopause with a subsequent reconnection in the distant magnetotail sets off a global convection cycle of the magnetic flux from the dayside into the magnetotail and then back to the dayside magnetosphere. Known as the Dungey cycle ${ }^{1}$, it gives rise to a wide range of global processes including magnetospheric substorms ${ }^{2}$, the buildup of storm-time ring current ${ }^{3}$, field-aligned current generation ${ }^{4}$, and variability of radiation belt intensities ${ }^{5}$.

A textbook picture of the magnetospheric convection invokes a large-scale duskward electric field, induced by the solar wind-magnetosphere coupling, which drives the Earthward bulk flow of cold plasma across the entire extent of the central plasma sheet ${ }^{6}$. As the plasma is convected towards the planet, into the regions of higher magnetic field intensity, it exhibits adiabatic energization in accordance with the conservation of the first two adiabatic invariants of the charged particle motion. In a quasi-dipolar geomagnetic field the inward convection produces energization predominantly in the direction perpendicular to the ambient magnetic field ${ }^{7}$, which, as the particles reach the inner magnetosphere and are energized to $10-100 \mathrm{keV}$, provides the free energy for EMIC and whistler wave growth ${ }^{8}$.

A growing wealth of observational evidence and physics-based modeling over past two decades suggest that plasma convection from the distant magnetotail to the inner magnetosphere occurs largely in the form of mesoscale flows, i.e., discrete enhancements in the Earthward plasma flow localized to a few Earth radii in the azimuthal direction ${ }^{9-11}$. Being typically preceded by sharp dipolarizations of magnetic field, the mesoscale convection flows are often referred to as dipolarization fronts or dipolarizing flux bundles ${ }^{12,13}$. To elucidate the fact that mesoscale flows are associated with localized dipolarizations 
of the ambient magnetic field, they are further referred to as dipolarization flows in this paper. Dipolarization flows provide an effective mechanism of particle transport and acceleration ${ }^{14,15}$. During storms, mesoscale dipolarization flows produce ion and electron injections into the heart of the ring current, which can account for much of its energy density ${ }^{16,17}$.

Recent observational studies provided compelling evidence that the mesoscale convection may also be the energy source of a wide range of kinetic processes ${ }^{18}$ such as kinetic Alfvén waves ${ }^{19}$, whistler chorus waves ${ }^{20}$, nonlinear time-domain structures $^{21}$, and shear-driven electron-ion hybrid modes at the leading edge of the flows ${ }^{22,23}$. In this paper we explore how the mesoscale convection produces velocity space instabilities in electron distributions, thus providing the pathway for the energy cascade from global to kinetic processes in Earth's magnetotail. We employ test-particle simulations in our Conservative Hamiltonian Integrator of Magnetospheric Particles (CHIMP) ${ }^{24}$ one way coupled to a high-resolution magnetohydrodynamic (MHD) simulations of plasma convection in the magnetotail. For the later we use the Lyon-Fedder-Mobarry (LFM) global magnetospheric model ${ }^{25}$. The next section describes results of test-particle simulations and the subsequent analysis of the anisotropy formation process they reveal. In the Discussion section, followed by Conclusions, we describe and discuss the comparison of our simulation results with the observations of electron instabilities and wave activity measured at the Magnetospheric Multiscale mission spacecraft around an isolated dipolarization flow.
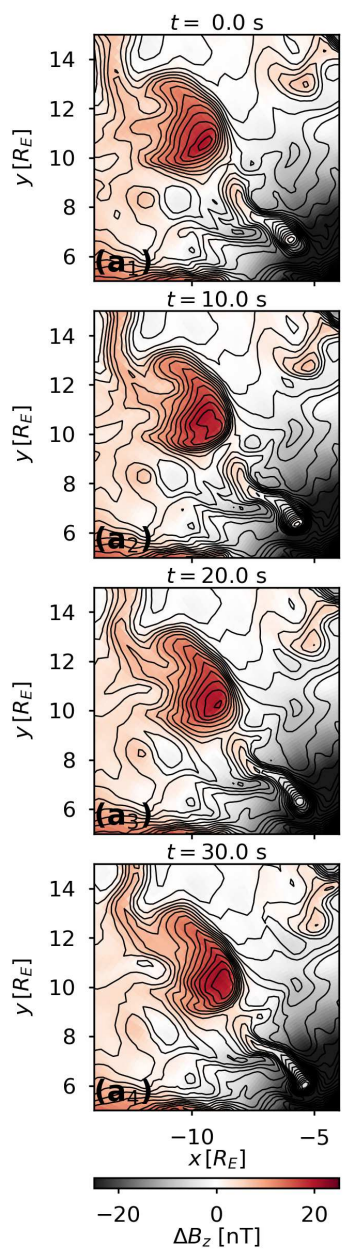
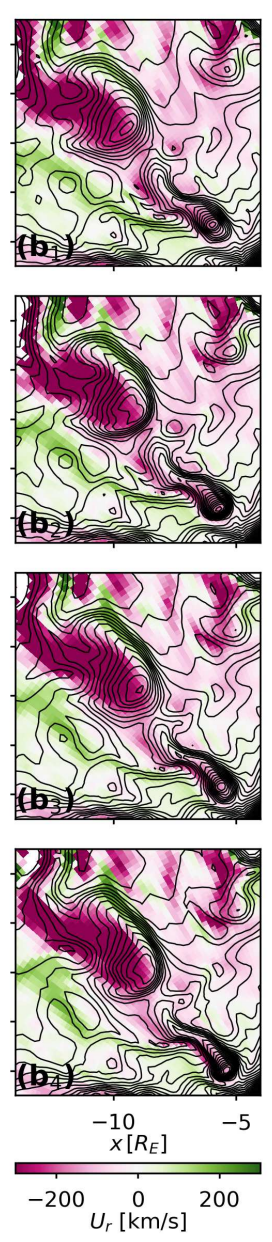
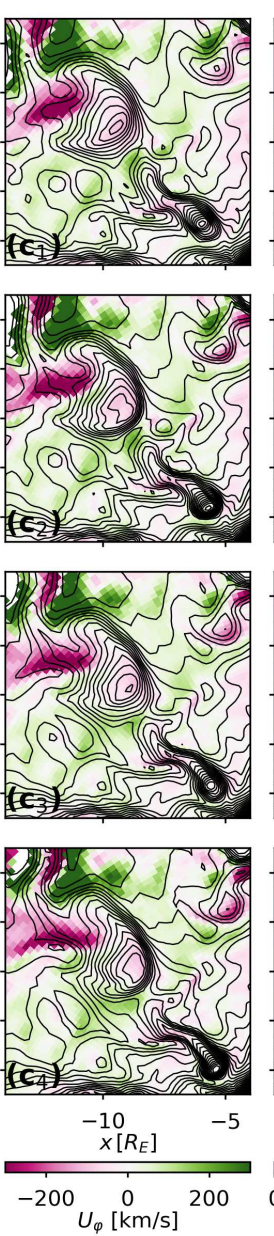
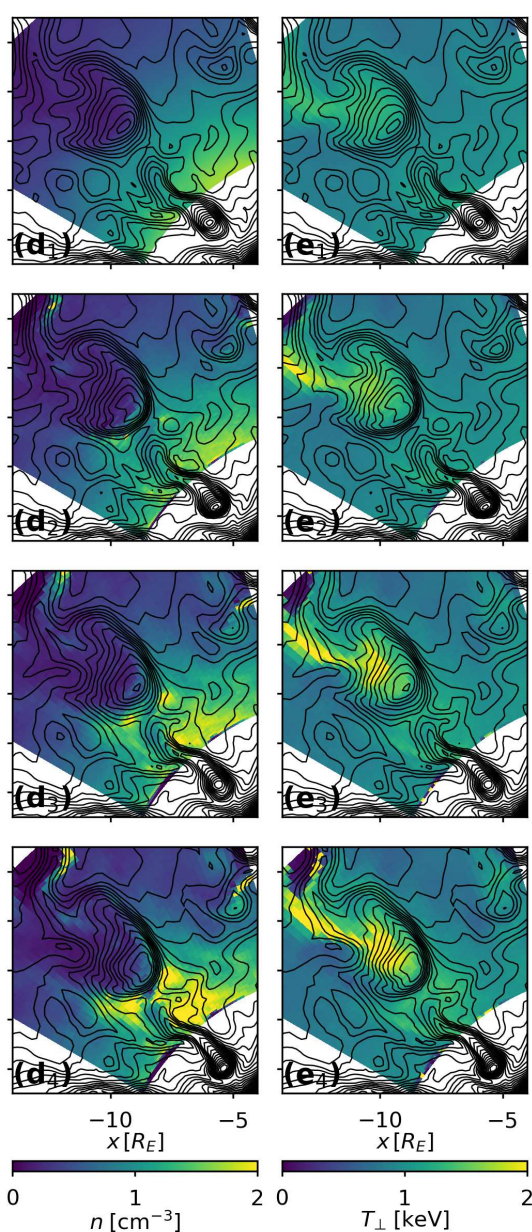
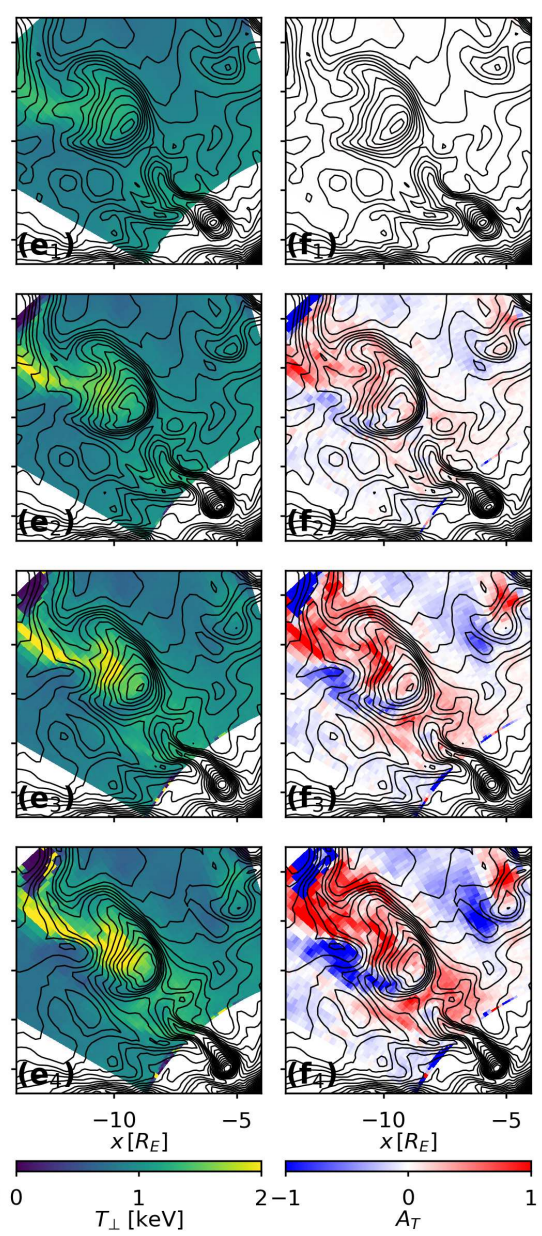

Figure 1. The growth of interchanging regions of parallel and perpendicular temperature anisotropies in electron distributions around a dipolarization flow in MHD and test-particle simulations. Electron PSD at $t=0$ were initialized with $T$ and $n$ from MHD simulations renormalized to a kappa distribution with $\kappa=3.5$ and $T_{i} / T_{e}=4$. Panels (a): external magnetic field, $\Delta B_{z}$; panels (b) and (c): the radial, $U_{r}$ and the azimuthal $U_{\varphi}$ components of the total equatorial drift velocity of $15 \mathrm{keV}$ electrons; panels (d) and (e) the number density, $n$, and perpendicular temperature $T_{\perp}$ of electrons in the test-particle simulations; panels (f): electron temperature anisotropy, $A_{T}$. Negative (positive) values of $A_{T}$ correspond to temperature anisotropies parallel (perpendicular) to the ambient magnetic field direction. The contours of constant total magnetic field intensity are shown with black lines in all panels of the figure. 


\section{Results}

\section{Anisotropy Formation at Dipolarization Front}

According to our previous analysis ${ }^{15,24}$, the magnetic terrain, carved by dipolarization flows in the ambient magnetic field plays a key role in the energization and transport of energetic particles from the magnetotail to the inner magnetosphere. Magnetic islands formed around dipolarization fronts can stably trap particles enabling their inward transport by over 10 Earth radii, $R_{e}$, leading to increases in their energy by more than an order of magnitude.

To explore how electron dynamics in a dipolarization flow channel impact the shape of the electron distribution function we conduct test-particle simulations with our Conservative Hamiltonian Integrator of Magnetospheric Particles (CHIMP) ${ }^{24}$. A three dimensional test-particle simulation was conducted in the electromagnetic field computed in the Lyon-Fedder-Mobarry (LFM) high-resolution global MHD magnetospheric mode ${ }^{25}$ during an interval of enhanced earthward convection ${ }^{11}$. At the simulation start, an ensemble of 40 million test-particles was initialized in an equatorial wedge centered at a large dipolarization front and distributed evenly over the entire range of the equatorial pitch angles from $0^{\circ}$ to $180^{\circ}$, and a wide energy range from $0.1 \mathrm{keV}$ to $200 \mathrm{keV}$ using a logarithmic spacing. Electron dynamics in the time varying electromagnetic field of the mesoscale convection were calculated in the guiding center approximation. Then, test-particle trajectories were used to compute the evolution of the electron phase space density under the assumption that initially particles had a kappa energy distribution and were fully isotropic in pitch angle (see Appendix A for details).

The results are summarized in Figure 1 showing simulation snapshots captured at a 10 second cadence along the magnetic equator. Figure 1a panels show the snapshots of the external magnetic field, $\Delta B_{z}$ (i.e., total magnetic field with Earth's dipolar field subtracted from it). Figure $1 \mathrm{~b}$ and Figure $1 \mathrm{c}$ contain the radial, $U_{r}$, and the azimuthal, $U_{\varphi}$ components of the combined $E \times B$ and gradient-curvature drift computed for $15 \mathrm{keV}$ electrons. Figure $1 \mathrm{~d}$ and Figure 1e depict the partial density, $n$, and perpendicular temperature, $T_{\perp}$, of electrons in our simulations, i.e., above the energy of $0.1 \mathrm{keV}$. Finally, Figure $1 \mathrm{f}$ panels show evolution of the temperature anisotropy, $A_{T}$, of the electron distribution computed as:

$$
A_{T}=\frac{T_{\perp}}{T_{\|}}-1, T_{\perp} \geq T_{\|} ; \text {or } A_{T}=-\frac{T_{\|}}{T_{\perp}}+1, T_{\perp}<T_{\|},
$$

where $T_{\perp}$ and $T_{\|}$are electron temperatures perpendicular and parallel to the magnetic field.

In all panels of Figure 1 contours of constant total magnetic field are shown with black lines. Closed contours indicate the magnetic islands associated with the dipolarization flows that enable stable trapping of energetic particles produced by a sharp gradient in the magnetic field at the interface of the dipolarization fronts embedded in the flows. As is shown in Figure 1f panels, dipolarization flows also lead to rapid emergence of interchanging regions of parallel and perpendicular temperature anisotropies. It took less that 20 seconds for the anisotropy values to grow above $\left|A_{T}\right|=1$ out of the initially isotropic pitch-angle distribution. While being a test-particle model CHIMP does not account for particle feedback onto the fields, it is reasonable to anticipate that in the real-world plasma such rapid surge of temperature anisotropies would quickly make electron distributions unstable to wave growth, thus providing the pathway for the energy cascade from mesoscale to kinetic structures.
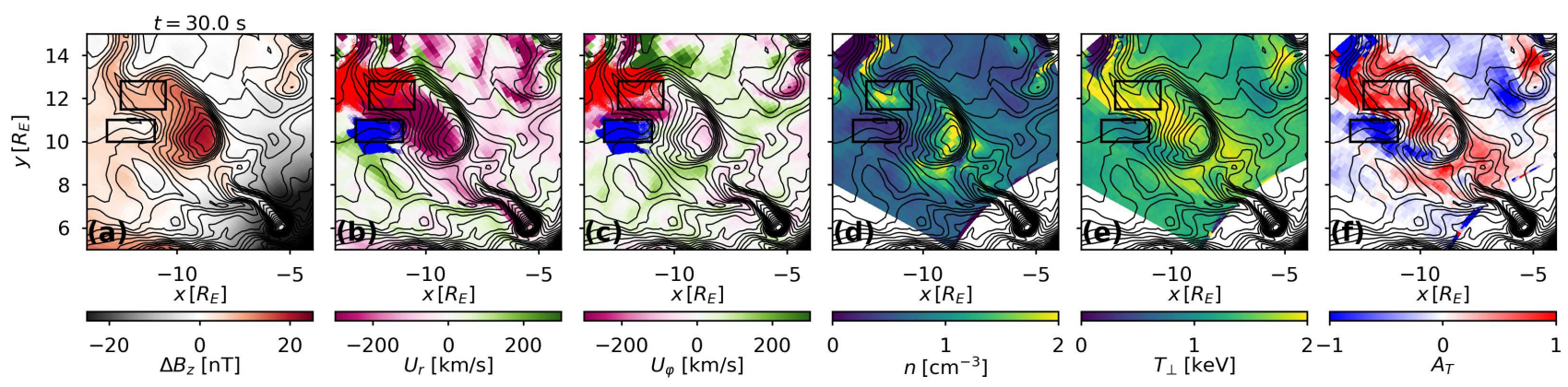

Figure 2. The snapshot of temperature anisotropies in the test-simulations (same as used in obtaining the results shown in Figure 1), re-initialized with an isotropic kappa distribution with $\kappa=3.5, T_{e}=1 \mathrm{keV}$, and $n_{T P}=1 \mathrm{~cm}^{-3}$, at the end of a $30 \mathrm{~s}$ simulation. Panels (a) and (b) show equatorial projections of test particle trajectories traced backward in time out of two regions of parallel (blue) and perpendicular (red) electron temperature anisotropies outlined with back rectangles in all the panels. 


\section{Path to Anisotropies}

Since the initial set up of the test-particle simulations shown in Figure 1 was derived from the MHD state with a pronounced mesoscale variations in both $n$ and $T$, the electron distribution, $f(\mathbf{r}, \alpha, K)$, started off with a builtin mesoscale structure both in $\mathbf{r}$ and in $K$, where $\mathbf{r}$ is the location in the equatorial plane, $\alpha$ is the equatorial value of the pitch angle, and $K$ is the kinetic energy. To isolate the process underlying the emergence of temperature anisotropies, we removed any builtin mesoscale structuring by re-initializing previous test-particle simulation a kappa distribution function isotropic in pitch angle and with $\kappa=-3.5, T=1$ $\mathrm{keV}$, and $n=1 \mathrm{~cm}^{-3}$, kept constant everywhere in the simulation domain. The results at the end of a 30 second simulation with the new phase space density initial conditions are summarized in Figure 2 in the format similar to Figure 1. As is evident from Figure $2 \mathrm{f}$, it exhibits strong pitch-angle anisotropies, with the pattern and the values similar to Figure $1 \mathrm{f}_{4}$.
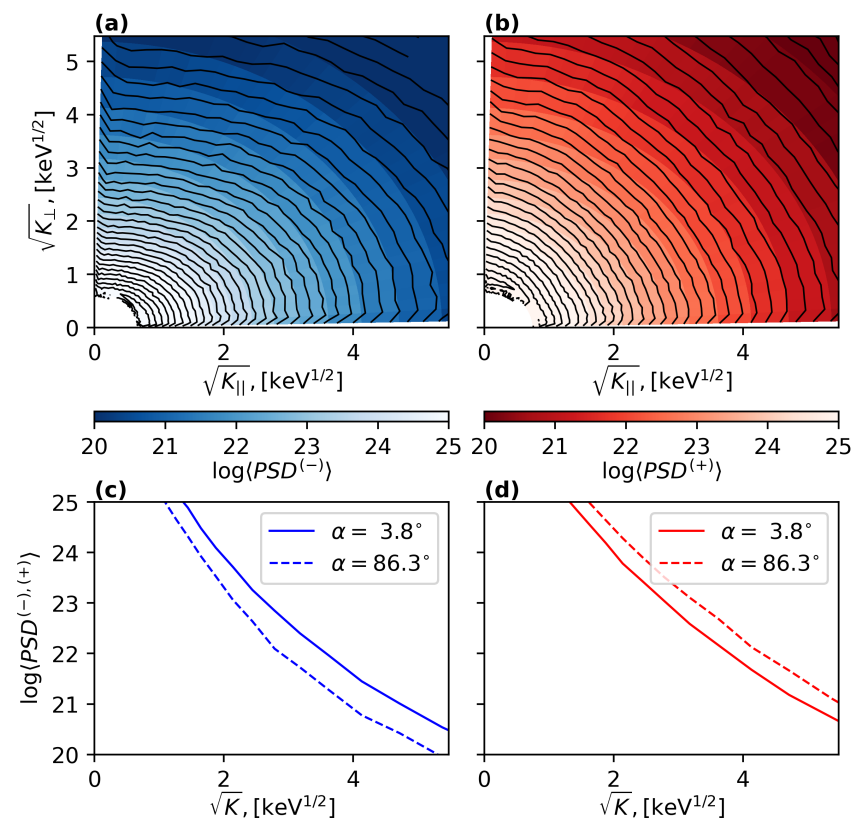

Figure 3. The parallel (panels (a) and (c)) and perpendicular (right panels (b) and (d)) anisotropies in electron distributions around dipolarization flows have similar values over the entire electron energy range. Panels (a) and (b) show electron PSD, averaged over the regions of parallel and perpendicular anisotropies shown in Figure 2. The line plots in panels (c) and (d) show PSD values at small and large pitch-angle values.

For further analysis, we used the anisotropy distribution at the end of the simulation interval (Figure $2 \mathrm{f}$ ) to select two regions with pronounced values of the parallel and perpendicular temperature anisotropies (marked with black rectangular outlines). Figure 3 shows details of the electron phase space density distributions averaged over the parallel (left panels) and the perpendicular (right panels) anisotropy regions. From the contour plots (top panels), and the line plots of the energy dependence of the electron distributions at small and large pitch-angle values (bottom panels), it follows that the anisotropies were produced by a process independent of electron energies, i.e., the anisotropy levels are similar over the entire energy range of the electron distribution. Considering that the perpendicular anisotropy emerged inside the inward dipolarization flow, whereas the parallel anisotropy formed outside of the dipolarization channel in the outward back flow, it is reasonable to assume that the anisotropies were attributed to the betatron effect.

To verify whether the emergence of interchanging patches of the parallel and perpendicular anisotropies is indeed controlled by the betatron effect, we selected large samples of test-particles from the final state of the simulations in the two selected anisotropy regions in Figure $3 f$ and followed their trajectories backward in time. To avoid tainting our consideration by energy dispersion in the gradient-curvature drift we considered electrons with the initial energy between 10 and $15 \mathrm{keV}$, which provide the largest contribution to temperature anisotropies. Distributions of the equatorial projections of the test particles from both ensembles are sown in Figure $2 b$ and $2 c$ with blue and red. As can be seen from the figure, the trajectories contributing to sculpting perpendicular and parallel temperature anisotropies, were separated by sharp features of the magnetic terrain around the dipolarization flow and hence did not overlap. The parallel anisotropy is formed by electrons drifting outward into the region of depleted magnetic field, whereas the perpendicular anisotropy is produced by electrons flowing inward towards higher magnetic field intensity.

Figure 4 shows the difference between the initial and the final states in the particle ensembles contributing to the parallel 

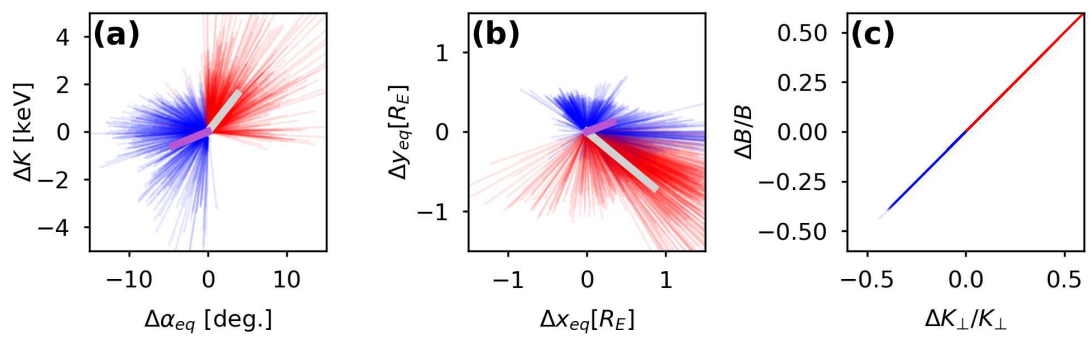

Figure 4. Over the course of the simulations, electron populations from the regions of parallel and perpendicular anisotropies, shown in Figure 2, do not overlap and undergo changes that point to the betatron affect as the mechanism of their generation. The figure shows the changes in the electron energy and pitch-angle (panel (a), equatorial location (panel (b)), and the equatorial values of the electron perpendicular energy and total magnetic field intensity at electron location (panel (c)) at the end of the $30 \mathrm{~s}$ simulation interval for particles in the regions of parallel (blue color) and perpendicular (red color) anisotropies.

(blue color) and the perpendicular (red color) anisotropies in the electron distribution. Parallel anisotropy was created by particles that (on average) lost their energy and decreased the equatorial pitch angle, whereas the perpendicular anisotropy was formed by particles that were (on average) energized and increased the pitch angle (Figure 4a). Figure 4b, which is showing the change in the particle equatorial location, well illustrates the absence of mixing between the two ensembles. Finally, the direct proportionality between the variations of the electron perpendicular energy and the changes in the magnetic field intensity in their locations, shown Figure 4c, confirms that the anisotropies were indeed formed due to the betatron effect.

\section{Discussion}

To examine whether the emergence of mesoscale patches of perpendicular and parallel anisotropies in the electron distribution function at dipolarization flows, as predicted by our test-particle simulations, does occur in Earth's magnetotail we use in situ observations from the Magnetospheric Multiscale (MMS) $\operatorname{mission}^{26}$ : the magnetic field from the Fluxgate Magnetometer ${ }^{27}$, plasma wave activity measured by the electric field probes ${ }^{28,29}$, end the electron data from Fast Plasma Investigation ${ }^{30}$. Figure 5 shows MMS3 observations of a dipolarization flow on 07/24/2017 at approximately $19.3 R_{E}$ in the magnetotail close to the midnight meridian. The dipolarization event was registered at approximately 12:49:30 UT, as is evident from a sharp increase in the magnetic field $B_{z}$ component (Figure 5c) along with an enhancement in the electron temperature (Figure 5a).

From the ratio of the electron perpendicular and parallel temperatures shown in Figure $5 \mathrm{~b}$ it follows that the electron distribution exhibited a parallel anisotropy ahead of the dipolarization flow front and a weak perpendicular anisotropy inside the flow. However, considering that any excess of free energy attributed to temperature anisotropies is expected to be quickly removed via generation of plasma waves, for further insight into the instabilities driven by the flow it is necessary to examine the wave activity associated with the event. Figure 5 shows a spectrogram of the electric field wave data (panel (d)), polarization angle (panel (e)), ellipticity (panel (f)), and the wave normal angle (panel (g)). As can be seen from Figure 5, the wave activity inside the dipolarization flow was comprised of intermittent bursts of whistler waves between approximately $100 \mathrm{and} 400 \mathrm{~Hz}$, propagating near-parallel to the ambient magnetic field, and broad-band wave activity, extending to above $1 \mathrm{kHz}$. With a typical flow speed of several hundred $\mathrm{km} / \mathrm{s}$, the spatial extent of the observed wave bursts is expected to be of the order of $\lesssim 1 R_{E}$, similar to the size of the anisotropy patches in our test-particle simulations.

The presence of intermittent bursts of whistler and broad-band wave activity observed in the dipolarization flow is fully consistent with a rapid growth of a mesoscale pattern of interchanging regions of the electron perpendicular and parallel temperature anisotropies as predicted in the test-particle simulations. Perpendicular temperature anisotropies are expected to produce parallel whistler mode instabilities ${ }^{8}$. Since the whistler instability is typically convective and the whistler group velocity is large, a remnant temperature anisotropy can remain alongside with the waves, as was the case shown in Figure $5 \mathrm{~b}$. The regions of parallel anisotropy can be a source to the firehose instability that can operate on time-scales of the order of the electron cyclotron frequency ${ }^{31}$ and produce the observed broad-band wave activity. Because the firehose instability is absolute and the modes it generates are non-propagating it is expected for the temperature anisotropy to be quickly relaxed, which would explain the absence of $T_{\|}>T_{\perp}$ inside the dipolarization flow in Figure 5b. Similarly, in the solar-wind the electron firehose instability is often used to explain the lack of observations with parallel temperature anisotropies. Two-dimensional simulations of the electron-firehose instability showed the temperature anisotropy quickly relaxed and left behind remnant whistler mode waves $^{32}$. 


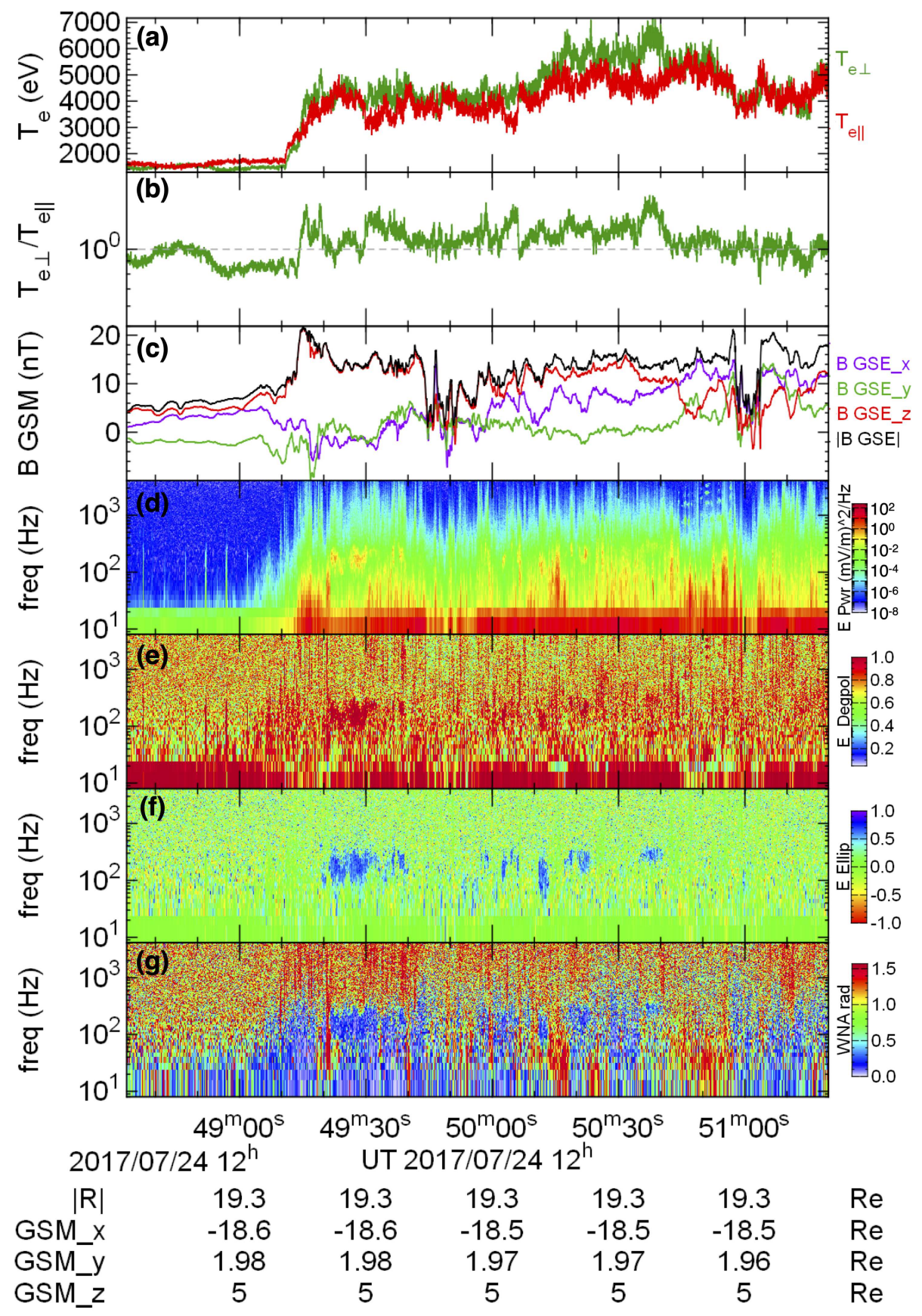

Figure 5. In situ observations of the wave activity and electron distribution in a dipolarization flow at MMS3 are fully consistent with the emergence of the mesoscale pattern of interchanging regions of parallel and perpendicular anisotropies predicted in our test particle simulations. Panel (a): electron temperature; panel (b): the ratio of the perpendicular and parallel temperatures; panel (c): magnetic field intensity; panel (d): electric field power spectral density; panel (e) electric field polarization; panel (f): electric field ellipticity, with negative (positive) values indicating left (right) hand polarization; panel (g): wave normal angle. 


\section{Conclusions}

In this study we used our one-way coupled global MHD and test-particle simulations to explore the growth of velocity-space anisotropies at Earthward propagating dipolarization flows, and then compared the simulation results with the in situ wave and plasma observations of an isolated dipolarization flow at the MMS3 spacecraft. Our conclusions, built on the earlier results ${ }^{15}$, further contribute to the paradigm shift in the magnetospheric convection as a complex cascade of coupled processes from global plasma instabilities and magnetic field reconfiguration, to mesoscale flows and dipolarization fronts, that power kinetic instabilities and wave structures:

- Earthward plasma convection in the magnetotail largely consists of mesoscale flows that exhibit complex magnetic terrain with features such as magnetic islands, which are instrumental to inward transport and energization of suprathermal particles.

- Mesoscale flows produce interchanging regions of parallel and perpendicular temperature anisotropies by polarizing electron distributions along the magnetic terrain boundaries with subsequent adiabatic heating and cooling (betatron effect).

- Rapid growth of mesoscale patches of the parallel and perpendicular temperature anisotropies in the electron distributions around dipolarization flows can provide free energy for the generation of broad-band and whistler waves reported from spacecraft observations.

\section{Methods}

\section{Test Particle Weighting}

To track global evolution of the particle phase space density (in this case electrons) we use a four-dimensional hyperplane corresponding to the magnetic equator: $\mathbf{X}=(r, \varphi, K, \alpha)$ with a volume element of $d \Gamma(\mathbf{X})=d^{3} p d V(r, \varphi, \alpha)$, where $r$ and $\varphi$ are the distance from the Earth's center and the azimuthal angle in the hyper plane of the magnetic equator defined by the magnetic field minima along the magnetic field lines, $K$ is the particle kinetic energy, $\alpha$ is the pitch angle, $p$ is the momentum, and $d V$ is the is the flux tube volume attached to the equatorial surface area of $r d r d \varphi$, which is accessible to a given pitch-angle particle:

$$
d V=B(r, \varphi, z=0) r d r d \varphi \int_{m}^{m^{\prime}} \frac{d l}{B}
$$

where the integration on the right side is carried between the conjugate bounce points of a particle with the pitch angle $\alpha$ above and below the magnetic equator.

The momentum space volume element is given by:

$$
d^{3} p=2 \pi \sin \alpha d \alpha \frac{\left(m c^{2}\right)^{2}}{c^{3}} \sqrt{\gamma^{2}-1} \gamma d K \simeq 2 \pi \sin \alpha d \alpha \sqrt{2} \frac{\left(m c^{2}\right)^{3 / 2}}{c^{2}} \sqrt{K} d K,
$$

where the approximate equality on the right hand side holds in a non-relativistic limit, $c$ is the speed of light, $\gamma$ is the relativistic factor, and $m$ is the rest mass. To initialize the electron phase space density we assume that at the simulation start the plasmasheet electron distribution is isotropic in pitch-angle and has a kappa distribution in energy ${ }^{33}$ :

$$
f(r, \varphi, K, \alpha)=\frac{4 \pi n_{0}}{\left(2 \pi m_{e} \kappa K_{0}\right)^{3 / 2}} \frac{\Gamma(\kappa+1)}{\Gamma(\kappa-1 / 2)}\left(1+\frac{K}{\kappa K_{0}}\right)^{-(1+\kappa)},
$$

with $K_{0}=T_{0}(1-3 / 2 \kappa)$, the plasma density $n_{0}=n_{M H D}(r, \varphi, t=0)$ and temperature $T_{0}=\frac{1}{4} T_{M H D}(r, \varphi, t=0)$ given by the global MHD simulations, and $\kappa=3.5$ in accordance with statistical properties of the plasmasheet dipolarization flows at 15 $R_{E}{ }^{34}$.

At the simulation start, $t=0$, each test particle in a given cell of the phase space grid, $\left\{\mathbf{X}^{n}\right\}$, is assigned with a weight according to the ratio of the number of the "real" particles, $f \delta \Gamma$, to the number of test-particles, $N_{m}$, in this grid cell:

$$
\begin{gathered}
w_{m}=\frac{1}{N_{m}} f\left(t=0, \mathbf{X}^{n}\right) \delta \Gamma\left(\mathbf{X}^{n}\right), N_{m}=\# \mathscr{I} \\
\mathscr{I}=\left\{\mathbf{X}_{m}(t=0): \mathbf{X}_{m} \in\left[\mathbf{X}^{n}, \mathbf{X}^{n}+\delta \mathbf{X}^{n}\right)\right\}
\end{gathered}
$$

Global evolution of the electron phase space density at $t>0$ is then computed from particle weights as:

$$
\begin{aligned}
& f\left(t>0, \mathbf{X}^{n}\right)=\frac{1}{\delta \Gamma\left(\mathbf{X}^{n}\right)} W_{\sigma}\left(\mathbf{X}^{n}\right) ; W_{\sigma}\left(\mathbf{X}^{n}\right)=\sum_{m=1}^{N_{m}} w_{m}, N_{m}=\# \mathscr{J} \\
& \mathscr{J}=\left\{\mathbf{X}_{m}(t>0): \mathbf{X}_{m} \in\left[\mathbf{X}^{n}, \mathbf{X}^{n}+\delta \mathbf{X}^{n}\right)\right\}
\end{aligned}
$$




\section{References}

1. Dungey, J. W. Interplanetary magnetic field and the auroral zones. Phys. Rev. Lett. 6, 47-48, DOI: 10.1103/physrevlett.6.47 (1961).

2. McPherron, R. L. Magnetospheric substorms. Rev. Geophys. 17, 657-681, DOI: https://doi.org/10.1029/RG017i004p00657 (1979).

3. Daglis, I. A., Thorne, R. M., Baumjohann, W. \& Orsini, S. The terrestrial ring current: Origin, formation, and decay. Rev. Geophys. 37, 407-438, DOI: https://doi.org/10.1029/1999RG900009 (1999).

4. Cowley, S. W. H. TUTORIAL: Magnetosphere-Ionosphere Interactions: A Tutorial Review. Wash. DC Am. Geophys. Union Geophys. Monogr. Ser. 118, 91, DOI: 10.1029/GM118p0091 (2000).

5. Dungey, J. W. The Effect of Quasi-Static Electric Fields on Van Allen Particles. 68, 3540-3541, DOI: 10.1029/ JZ068i011p03540 (1963).

6. Akasofu, S. I. The solar wind-magnetosphere dynamo and the magnetospheric substorm. 23, 817-823, DOI: 10.1016/ 0032-0633(75)90018-5 (1975).

7. Schulz, M. \& Lanzerotti, L. J. Particle Diffusion in the Radiation Belts, vol. 7 of Physics and Chemistry in Space (Springer-Verlag, Berlin, Heidelberg, New York, 1974).

8. Kennel, C. F. \& Petscheck, H. E. Limit on stably trapped particle fluxes. J. Geophys. Res. 71, 427 (1966).

9. Angelopoulos, V. et al. Magnetotail flow bursts: association to global magnetospheric circulation, relationship to ionospheric activity and direct evidence for localization. Geophys. Res. Lett. 24, 2271 (1997).

10. Nakamura, R. et al. Spatial scale of high-speed flows in the plasma sheet observed by Cluster. Geophys. Res. Lett. 31, L09804, DOI: doi:10.1029/2004GL019558 (2004).

11. Wiltberger, M., Merkin, V., Lyon, J. G. \& Ohtani, S. High-resolution global magnetohydrodynamic simulation of bursty bulk flows. J. Geophys. Res. 120, 4555, DOI: 10.1002/2015JA021080 (2015).

12. Runov, A. et al. THEMIS observations of an earthward-propagating dipolarization front. Geophys. Res. Lett. 36, L14106, DOI: doi:10.1029/2009GL038980 (2009).

13. Gabrielse, C., Harris, C., Angelopoulos, V., Artemyev, A. \& Runov, A. The role of localized inductive electric fields in electron injections around dipolarizing flux bundles. J. Geophys. Res. Space Phys. 121, 9560-9585, DOI: https: //doi.org/10.1002/2016JA023061 (2016).

14. Mitchell, D. et al. Charge-dependent energization in substorm ion injections in the middle and outer magnetosphere: Observations and a simple model. Geophys. Res. Lett. in preparation (2016).

15. Ukhorskiy, A. Y. et al. Ion trapping and acceleration at dipolarization fronts: High-resolution mhd and test-particle simulations. J. Geophys. Res. Space Phys. 123, 5580-5589, DOI: https://doi.org/10.1029/2018JA025370 (2018).

16. Gkioulidou, M. et al. The role of small-scale ion injections in the buildup of Earth's ring current pressure: Van Allen Probes observations of the 17 March 2013 storm. J. Geophys. Res. 119, 7327, DOI: 10.1002/2014JA020096 (2014).

17. Sorathia, K. A. et al. The role of mesoscale plasma sheet dynamics in ring current formation. Front. Astron. Space Sci. 8, 192, DOI: 10.3389/fspas.2021.761875 (2021).

18. Malaspina, D. M. et al. Electric field structures and waves at plasma boundaries in the inner magnetosphere. J. Geophys. Res. Space Phys. 120, 4246-4263, DOI: https://doi.org/10.1002/2015JA021137 (2015).

19. Chaston, C. C. et al. Broadband low-frequency electromagnetic waves in the inner magnetosphere. J. Geophys. Res. Space Phys. 120, 8603-8615, DOI: https://doi.org/10.1002/2015JA021690 (2015).

20. Malaspina, D. M. et al. Nonlinear electric field structures in the inner magnetosphere. Geophys. Res. Lett. 41, 5693-5701, DOI: https://doi.org/10.1002/2014GL061109 (2014).

21. Mozer, F. S. et al. Time domain structures: What and where they are, what they do, and how they are made. Geophys. Res. Lett. 42, 3627-3638, DOI: https://doi.org/10.1002/2015GL063946 (2015).

22. Ganguli, G. et al. Kinetic equilibrium of dipolarization fronts. Sci. Reports 8, 17186, DOI: 10.1038/s41598-018-35349-9 (2018).

23. Fletcher, A. C. et al. Kinetic equilibrium and stability analysis of dipolarization fronts. J. Geophys. Res. Space Phys. 124, 2010-2028, DOI: 10.1029/2018ja026433 (2019). 
24. Sorathia, K. A., Merkin, V. G., Ukhorskiy, A. Y., Mauk, B. H. \& Sibeck, D. G. Energetic particle loss through the magnetopause: A combined global MHD and test-particle study. J. Geophys. Res. 122, 9329, DOI: 10.1002/2017JA024268 (2017).

25. Lyon, J. G., Fedder, J. A. \& Mobarry, C. M. The Lyon-Fedder-Mobarry (LFM) global MHD magnetospheric simulation code. J. Atmos. Solar-Terr. Phys. 25, 3039 (2004).

26. Burch, J. L., Moore, T. E., Torbert, R. B. \& Giles, B. L. Magnetospheric Multiscale Overview and Science Objectives. Space Sci. Rev. 199, 5-21, DOI: 10.1007/s11214-015-0164-9 (2016).

27. Russell, C. T. et al. The Magnetospheric Multiscale Magnetometers. Space Sci. Rev. 199, 189-256, DOI: $10.1007 /$ s11214-014-0057-3 (2016).

28. Lindqvist, P.-A. et al. The Spin-Plane Double Probe Electric Field Instrument for MMS. Space Sci. Rev. 199, 137-165, DOI: $10.1007 / \mathrm{s} 11214-014-0116-9$ (2016).

29. Ergun, R. E. et al. The Axial Double Probe and Fields Signal Processing for the MMS Mission. Space Sci. Rev. 199, 167-188, DOI: 10.1007/s11214-014-0115-x (2016).

30. Pollock, C. et al. Fast Plasma Investigation for Magnetospheric Multiscale. Space Sci. Rev. 199, 331-406, DOI: 10.1007/s11214-016-0245-4 (2016).

31. Li, X. \& Habbal, S. R. Electron kinetic firehose instability. J. Geophys. Res. 105, 27,377-27,386 (2000).

32. Camporeale, E. \& Burgess, D. Electron firehose instability: Kinetic linear theory and two dimensional particle-in-cell simulations. J. Geophys. Res. 113, 107107 (2008).

33. Christon, S., Williams, D. J., Mitchell, D. G., Frank, L. A. \& Huang, C. Y. Spectral characteristics of plasma sheet ion and electron populations during undisturbed geomagnetic conditions. J. Geophys. Res. Space Phys. 94, 13409-13424, DOI: https://doi.org/10.1029/JA094iA10p13409 (1989).

34. Runov, A. et al. Average thermodynamic and spectral properties of plasma in and around dipolarizing flux bundles. $J$. Geophys. Res. 120, 4369, DOI: 10.1002/2015JA021166 (2015).

\section{Acknowledgements}

MMS data can be found at the MMS public Science Data Center (https: / / lasp. colorado. edu/mms/sdc/public/). This work was supported by NASA MMS contracts to the instrument teams. The research was supported by NASA grants NNX16AG73G, 80NSSC17K0679, 80NSSC19K0241, NNX17AB81G, NNH17AE70I and Naval Research Laboratory Base Program.

\section{Author contributions statement}

A.U., K.S., and V.M. conceived and conducted numerical simulations, C.C. and A.F. conducted wave growth analysis, D.M. and S.S. carried out MMS data analysis. All authors reviewed the manuscript. 